\title{
Elucidation of the Role of Constraints in Economic Model Predictive Control
}

\author{
Matthew Ellis ${ }^{\mathrm{a}}$, Helen Durand ${ }^{\mathrm{a}}$, Panagiotis D. Christofides ${ }^{*, a, b}$ \\ ${ }^{a}$ Department of Chemical and Biomolecular Engineering, University of California, Los Angeles, \\ CA, 90095-1592, USA. \\ ${ }^{b}$ Department of Electrical Engineering, University of California, Los Angeles, CA 90095-1592, \\ USA.
}

\section{Abstract}

Economic model predictive control (EMPC) is a predictive feedback control methodology that unifies economic optimization and control. EMPC uses a stage cost that reflects the process/system economics. In general, the stage cost used is not a quadratic stage cost like that typically used in standard tracking model predictive control. In this paper, a brief overview of EMPC methods is provided. In particular, the role of constraints imposed in the optimization problem of EMPC for feasibility, closed-loop stability, and closed-loop performance is explained. Three main types of constraints are considered including terminal equality constraints, terminal region constraints, and constraints designed via Lyapunov-based techniques. The paper closes with a well-known chemical engineering example (a non-isothermal CSTR with a second-order reaction) to illustrate the effectiveness of time-varying operation to improve closed-loop economic performance compared to steady-state operation and to demonstrate the impact of economically

\footnotetext{
*Corresponding author: Tel: +1 (310) 794-1015; Fax: +1 (310) 206-4107; E-mail: pdc@seas.ucla.edu.
} 
motivated constraints on optimal operation.

Key words: Economic model predictive control, process economic optimization, process control, nonlinear systems

\section{Introduction}

Economic model predictive control (EMPC) has attracted significant attention and research over the last five years. This interest is a result of the ability of EMPC to integrate optimization of process economics with process control by incorporating a general stage cost function in the optimization problem and allowing for consistently dynamic (time-varying) process operation without requiring the process to settle at a steady-state or reference trajectory (Helbig et al. (2000); Engell (2007); Rawlings and Amrit (2009); Amrit et al. (2011); Angeli et al. (2012); Huang et al. (2011); Heidarinejad et al. (2012a); see, also, the reviews Rawlings et al. (2012); Ellis et al. (2014) for a more complete overview and reference list of the EMPC literature). In contrast to tracking model predictive control (MPC), which usually incorporates a quadratic stage cost, the stage cost of EMPC is chosen as a direct or indirect measure of the process/system economic performance. As a result of the general stage cost used, EMPC may force a process to operate in a time-varying manner to optimize the economics. The rigorous design of EMPC schemes that operate large-scale processes in a dynamically optimal fashion while maintaining stability of the closed-loop system is challenging because traditional stability analysis concepts, such as asymptotic stability of a steady-state for a process under a given controller, may be inapplicable to a closed-loop system under EMPC.

To address the three key fundamental issues of feasibility, stability, and eco- 
nomic performance, constraints are often employed in the EMPC problem formulation. To this end, many EMPC formulations have been proposed encompassing theoretical analysis of closed-loop properties (e.g., Ferramosca et al. (2010); Amrit et al. (2011); Angeli et al. (2012); Huang et al. (2011, 2012); Heidarineiad et al. (2012a); Grüne (2013); Alessandretti et al. (2014); Bayer et al. (2014); Faulwasser et al. (2014); Grüne and Stieler (2014); Limon et al. (2014); Müller et al. (2014a); Zavala (2015)), optimization and computational issues (e.g., Kadam and Marquardt (2007); Würth and Marquardt (2014); Biegler et al. (2015)), and implementation and applications (e.g., Heidarineiad et al. (2012b); Omell and Chmielewski (2013); Grosso et al. (2014); Zhang et al. (2014); Ellis and Christofides (2015b)).

This article describes the role and implications of constraints used in EMPC. It is an extended version of the work Ellis and Christofides (2015a). Owing to space limitations, certain technical assumptions are omitted and statements of the results are summarized.

Notation: $|\cdot|$ denotes the Euclidean norm of a vector. The symbol $S(\Delta)$ denotes the family of piecewise constant functions with period $\Delta>0$. A continuous function $\beta: \mathbb{R}^{n} \rightarrow \mathbb{R}$ is positive definite if $\beta(0)=0$ and $\beta(x)>0$ for all $x \neq 0$. A continuous function $\alpha:[0, a) \rightarrow[0, \infty)$ belongs to class $\mathcal{K}$ if it strictly increasing and $\alpha(0)=0$.

\subsection{Class of Nonlinear Systems}

The class of systems considered is described by the system of nonlinear ordinary differential equations (ODEs):

$$
\dot{x}(t)=f(x(t), u(t), w(t))
$$


where $x(t) \in \mathbb{X} \subset \mathbb{R}^{n}$ denotes the state vector, $u(t) \in \mathbb{U} \subset \mathbb{R}^{m}$ denotes the manipulated (control) input vector, and $w(t) \in \mathbb{W} \subset \mathbb{R}^{l}$ denotes the disturbance vector. The set of admissible input values $\mathbb{U}$ is compact, and the disturbance vector is bounded in the set $\mathbb{W}:=\left\{w \in \mathbb{R}^{l}|| w \mid \leq \theta\right\}$ where $\theta>0$ bounds the norm of the disturbance vector. The vector function $f: \mathbb{X} \times \mathbb{U} \times \mathbb{W} \rightarrow \mathbb{X}$ is locally Lipschitz on $\mathbb{X} \times \mathbb{U} \times \mathbb{W}$. A state measurement is synchronously sampled at sampling instances denoted as $t_{k}:=k \Delta$ where $k \in \mathbb{I}_{\geq 0}$ and $\Delta>0$ is the sampling period (the initial time is taken to be zero). The assumption of state feedback is standard owing to the fact that the separation principle does not generally hold for nonlinear systems. Nevertheless, some rigorous output feedback implementations of EMPC exist (e.g., Heidarinejad et al. (2012b); Zhang et al. (2014)). The system (11) is equipped with a continuous function $l_{e}: \mathbb{X} \times \mathbb{U} \rightarrow \mathbb{R}$, which reflects the instantaneous process/system economics. The function $l_{e}(\cdot, \cdot)$ will be used as a stage cost in a model predictive control (MPC) framework and will be referred to as the economic stage cost. The system (1) may have additional constraints other than the input and state constraints. Collecting all the constraints including the input, state, and additional constraints, the constraints may be written generally as static constraints:

$$
g_{s}(x, u) \leq 0
$$

where $g_{s}: \mathbb{X} \times \mathbb{U} \rightarrow \mathbb{R}^{n_{s}}$ and as dynamic constraints (e.g., average constraints):

$$
\int_{0}^{t_{d}} g_{d}(x(t), u(t)) d t \leq 0
$$


where $g_{d}: \mathbb{X} \times \mathbb{U} \rightarrow \mathbb{R}^{n_{d}}$ and $t_{d}$ is the time horizon that the constraint is imposed. The dynamic constraints are often motivated by economic considerations. The economically optimal steady-state and steady-state input pair is:

$$
\left(x_{s}^{*}, u_{s}^{*}\right)=\underset{\left(x_{s}, u_{s}\right)}{\arg \min }\left\{l_{e}\left(x_{s}, u_{s}\right): \begin{array}{c}
f\left(x_{s}, u_{s}, 0\right)=0 \\
g_{s}\left(x_{s}, u_{s}\right) \leq 0 \\
g_{d}\left(x_{s}, u_{s}\right) \leq 0
\end{array}\right\} .
$$

With the notation above, the optimal steady-state pair $\left(x_{s}^{*}, u_{s}^{*}\right)$ is assumed to be unique. If the minimizing pair is not unique, let $\left(x_{s}^{*}, u_{s}^{*}\right)$ denote one of the minimizing steady-state pairs. The optimal steady-state is taken to be the origin of the unforced system $(f(0,0,0)=0)$.

Remark 1. Time-varying economic considerations such as customer demand changes, dynamic energy pricing, and variable feedstock quality may lead to explicitly time-varying economic stage costs as well as time-dependent economic-oriented constraints. While economic stage costs that are not explicitly time-dependent are considered here, some EMPC methodologies exist for handling some issues related to time-varying economic stage costs such as a Lyapunov-based EMPC formulation that allows for changing regions of operation as the economic stage cost changes with time while guaranteeing closed-loop stability (Ellis and Christofides (2014a)). Another potentially useful concept that may help enable EMPC to handle time-varying economic stage costs is the use of a generalized terminal constraint or self-tuning terminal region and terminal cost (e.g., Ferramosca et al. (2010); Fagiano and Teel (2013); Müller et al. (2014a)). 


\section{EMPC Schemes: Feasibility, Closed-loop Stability, and Performance}

Economic model predictive control is an MPC method that uses the economic stage cost in its formulation. The EMPC problem, with a finite-time prediction horizon, can be broadly characterized by the following optimal control problem (OCP):

$$
\begin{array}{cl}
\min _{u(\cdot) \in S(\Delta)} & \int_{t_{k}}^{t_{k+N}} l_{e}(\tilde{x}(t), u(t)) d t+V_{f}\left(\tilde{x}\left(t_{k+N}\right)\right) \\
\text { s.t. } \quad & \tilde{\tilde{x}}(t)=f(\tilde{x}(t), u(t), 0) \\
& \tilde{x}\left(t_{k}\right)=x\left(t_{k}\right) \\
& g_{s}(\tilde{x}(t), u(t)) \leq 0, \forall t \in\left[t_{k}, t_{k+N}\right] \\
& \int_{t_{k}}^{t_{k+N}} g_{d}(\tilde{x}(t), u(t)) d t \leq 0
\end{array}
$$

where the decision variable of the optimization problem is the piecewise constant input trajectory over the prediction horizon (i.e., the time interval $\left[t_{k}, t_{k+N}\right)$ ) and $\tilde{x}$ denotes the predicted state trajectory over the prediction horizon. Higher order control parameterizations may also be considered. Nevertheless, sample-and-hold (i.e., zeroth-order hold) implementation of controls is one of the most commonly employed control parameterizations (i.e., $u(\cdot) \in S(\Delta)$ as in (5a)).

The cost functional (5a) consists of the economic stage cost with a terminal cost/penalty $V_{f}: \mathbb{X} \rightarrow \mathbb{R}$. The nominal dynamic model (5b) is used to predict the future evolution of the system and is initialized with a state measurement $(5 \mathrm{c})$. When available, disturbance estimates or predictions may be incorporated in the model (5b). The constraints (5d)-(5e) represent the system constraints which may include input, state, mixed state and input, economic, and stability constraints. 
The constraint (5e) may be time-varying (i.e., formulated for the sampling time $t_{k}$, so that the constraint (3) is satisfied over the desired operating interval). With slight abuse of notation, (5e) is not necessarily the same as (3). For the remainder of this section, the dynamic constraints are dropped and only EMPC schemes of the form (5a)-(5d) are considered, except for a brief discussion of the impact of dynamic constraints on the trajectories of EMPC with input rate of change constraints. Thus, the constraint set is $\mathbb{Z}:=\left\{(x, u): x \in \mathbb{X}, u \in \mathbb{U}, g_{s}(x, u) \leq\right.$ $0\} \subseteq \mathbb{X} \times \mathbb{U}$ and $\mathbb{Z}$ is assumed to be compact.

Like tracking MPC, EMPC is typically implemented with a receding horizon implementation to better approximate the infinite horizon solution and to ensure robustness of the control solution to disturbances and open-loop instabilities. At a sampling time $t_{k}$, the EMPC receives a state measurement, which is used to initialize the model (5b). The OCP (5) is solved on-line for a (local) optimal piecewise input trajectory, denoted by $u^{*}\left(t \mid t_{k}\right)$ for $t \in\left[t_{k}, t_{k+N}\right)$. The control action computed for the first sampling period of the prediction horizon, denoted as $u^{*}\left(t_{k} \mid t_{k}\right)$, is sent to the control actuators to be implemented over the sampling period from $t_{k}$ to $t_{k+1}$ (i.e., sample-and-hold implementation). At the next sampling time, the OCP (5) is re-solved after receiving a new state measurement and by shifting the prediction horizon into the future by one sampling period.

EMPC, which consists of the on-line solution of the OCP (5) along with a receding horizon implementation, results in an implicit state feedback law $u(t)=$ $\kappa\left(x\left(t_{k}\right)\right)$ for $t \in\left[t_{k}, t_{k+1}\right)$. From a theoretical perspective, three fundamental issues are considered and addressed with respect to EMPC. The first consideration 
is the feasibility of the optimization problem (both initial and recursive feasibility may be considered). Second, if (5) is recursively feasible, it is important to consider the stability properties of the closed-loop system under EMPC. In general, one may not expect that EMPC will force the state to a desired steady-state. The last theoretical consideration is closed-loop economic performance under EMPC. Within the context of EMPC, closed-loop performance typically means the average closed-loop economic performance. Over a finite-time operating interval of length $t_{f}$, the average performance is defined by the following index:

$$
\bar{J}_{e}:=\frac{1}{t_{f}} \int_{0}^{t_{f}} l_{e}(x(t), u(t)) d t
$$

where $x$ and $u$ are the closed-loop state and input trajectories, respectively, and over an infinite-time operating interval, the infinite-time (asymptotic) average economic performance is given by:

$$
\bar{J}_{e, \infty}:=\limsup _{t_{f} \rightarrow \infty} \frac{1}{t_{f}} \int_{0}^{t_{f}} l_{e}(x(t), u(t)) d t .
$$

Without additional assumptions and conditions, one can easily construct examples of systems and stage costs of the form described above where the closedloop under EMPC (without additional stability constraints) is unstable. Clearly, additional conditions and/or constraints enforced in the EMPC problem may be needed to guarantee closed-loop stability. Some theoretical investigations on EMPC that do not incorporate additional stability constraints exist including the work of Grüne (2013); Grüne and Stieler (2014) which require that the resulting EMPC has a sufficiently long horizon as well as that certain controllability assumptions and turnpike conditions be satisfied to guarantee closed-loop stability 
and performance properties. Moreover, even though EMPC optimizes the process/system economics, it does so over a finite-time prediction horizon. Over long periods of operation, no conclusion, in general, can be made on closed-loop performance under EMPC (without additional constraints). For provable results on feasibility, closed-loop stability, and closed-loop performance under EMPC, typically, additional stability and/or performance constraints are added to the formulation of EMPC. These formulations are discussed in the subsequent sections.

Remark 2. It is important to clarify why the average economic performance is of interest for EMPC. EMPC may dictate a time-varying operating strategy to optimize the process/system economics. Thus, it may not enforce convergence to the economically optimal steady-state. While the instantaneous stage cost under EMPC at any time may be better or worse than the stage cost at the economically optimal steady-state and steady-state input pair, the average economic performance under the time-varying operating policy dictated by EMPC over the length of operation may be better than that achieved by operation at the economically optimal steady-state. More explanation on this point is provided below where an example in which time-varying operation achieves better performance compared to steady-state operation is considered.

Remark 3. To address closed-loop stability, one may consider employing an infinite horizon in the EMPC. This may be a more appropriate prediction horizon because many chemical processes are continuously operated over long periods of time (practically infinite time). At least intuitively, the resulting control law 
will provide some form of closed-loop stability assuming the existence of a solution to the infinite horizon EMPC as well as the ability to solve for a solution on-line. However, it is difficult to solve an OCP with an infinite horizon. To overcome this problem, two approaches include: (1) approximating the infinite horizon with a sufficiently long finite-time horizon and (2) dividing the infinite horizon into a finite-time horizon and estimating the infinite horizon tail through an auxiliary control law or with modeling-based techniques (e.g., Diehl et al. (2011); Huang et al. (2012); Omell and Chmielewski (2013)). Although some of these EMPC schemes may be computationally tractable, the use of constraints typically enables shorter prediction horizons reducing the on-line computation relative to those that require sufficiently long horizons. Thus, infinite horizon EMPC and EMPC without stability constraints are not discussed, but rather, EMPC systems formulated with constraints to provide guaranteed closed-loop properties are considered.

\subsection{EMPC with an Equality Terminal Constraint}

Much of the recent theoretical work on EMPC investigates the extension of stabilizing elements used in tracking MPC to EMPC such as adding a terminal constraint and/or terminal cost (see, for instance, Mayne et al. (2000) for more details on the use of terminal constraints and/or a terminal cost within the context of tracking MPC). Numerous EMPC formulations and theoretical developments which include a terminal constraint and/or terminal cost have been proposed and studied (e.g., Amrit et al. (2011); Diehl et al. (2011); Huang et al. (2011); Angeli et al. (2012); Müller and Allgöwer (2012)). There are two main types of 
EMPC with terminal constraints: (1) EMPC with an equality terminal constraint, and (2) EMPC with a terminal region constraint. In this section, the former type of EMPC is considered which is an EMPC (5a)-(5d) with the following constraint:

$$
\tilde{x}\left(t_{k+N}\right)=x_{s}^{*} .
$$

The constraint (8) forces the predicted state trajectory to converge to the optimal steady-state at the end of the finite-time horizon. For EMPC with an equality terminal constraint, the terminal cost is often omitted as it is not required for stability and performance guarantees.

\subsubsection{Feasibility.}

EMPC with a terminal equality constraint is (initially) feasible for any initial state in $\mathbb{X}_{N} \in \mathbb{R}^{n}$ which denotes the feasible region of EMPC (5a)-(5d), (8). The feasible region depends on the prediction horizon, and an explicit characterization of $\mathbb{X}_{N}$ is difficult in general. Recursive feasibility (i.e., feasibility at each subsequent sampling time) of EMPC with an equality terminal constraint is guaranteed for the nominally operated system for any initial state $x(0) \in \mathbb{X}_{N}$. This follows from the fact that a feasible solution to the EMPC can be constructed from the solution from the previous sampling time. Namely, $u(t)=u^{*}\left(t \mid t_{k-1}\right)$ for $t \in\left[t_{k}, t_{k+N-1}\right)$ and $u(t)=u_{s}^{*}$ for $t \in\left[t_{k+N-1}, t_{k+N}\right)$ is a feasible solution for the EMPC at $t_{k}$ because it satisfies the constraints and the terminal constraint (8). However, recursive feasibility is harder to show, in general, when $w(\cdot) \not \equiv 0$. 


\subsubsection{Closed-loop Stability.}

With respect to closed-loop stability, a weak notion of stability follows from the EMPC with terminal constraint formulation. If the initial state is in the feasible set, the closed-loop state trajectory remains contained in the feasible set for nominal operation. For stronger stability properties (e.g., asymptotic stability of $x_{s}^{*}$, additional assumptions on the closed-loop system must be satisfied. To discuss this issue, nonlinear discrete-time systems are considered that have the form:

$$
x(k+1)=f_{d}(x(k), u(k))
$$

where $f_{d}: \mathbb{X} \times \mathbb{U} \rightarrow \mathbb{X}$ is the discrete-time state transition map and $k \in \mathbb{I}_{\geq 0}$ is the time index. As before, the system (9) is subject to mixed state and input constraints $(x, u) \in \mathbb{Z} \subseteq \mathbb{X} \times \mathbb{U}$ where $\mathbb{Z}$ is a compact set and the origin is assumed to be the optimal steady-state $\left(f_{d}(0,0)=0\right)$. Discrete-time systems are considered here to maintain consistency with the literature on the topic. Nonetheless, some of these conditions and results have been extended to continuous-time systems (e.g., Alessandretti et al. (2014)). The notion of dissipativity has been extended to EMPC which leads to stronger stability properties under EMPC with an equality terminal constraint.

Definition 1 (Angeli et al. (2012)). The system of (9) is strictly dissipative with respect to a supply rate $s: \mathbb{X} \times \mathbb{U} \rightarrow \mathbb{R}$ if there exist a function $\lambda: \mathbb{X} \rightarrow \mathbb{R}$ and a positive definite function $\beta: \mathbb{X} \rightarrow \mathbb{R}_{\geq 0}$ such that

$$
\lambda\left(f_{d}(x, u)\right)-\lambda(x) \leq-\beta(x)+s(x, u)
$$


for all $(x, u) \in \mathbb{Z}$.

If the system (9) is strictly dissipative with a supply rate:

$$
s(x, u)=l_{e}(x, u)-l_{e}\left(x_{s}^{*}, u_{s}^{*}\right)
$$

then, the optimal steady-state is asymptotically stable for the closed-loop system under EMPC with an equality terminal constraint (Angeli et al. (2012)). Moreover, a Lyapunov function for the closed-loop system was derived using the cost functional of the so-called rotated cost function Angeli et al. (2012):

$$
L(x, u):=l_{e}(x, u)+\lambda(x)-\lambda\left(f_{d}(x, u)\right) .
$$

The idea of using the rotated cost function to construct a Lyapunov function for the closed-loop system was originally proposed in Diehl et al. (2011). However, it relied on strong duality of the steady-state optimization problem, which is a stronger assumption than strict dissipativity.

\subsubsection{Closed-loop Performance.}

Utilizing the optimal input trajectory at $t_{k}$ (or time step $k$ in discrete-time) as a feasible solution to the EMPC at the next sampling period, one can upper bound the difference between the cost functional value at the next sampling time and at the current sampling time under nominal operation. The optimal input trajectory in discrete-time is denoted $u^{*}(j \mid k)$ for $j=k, k+1, \ldots, k+N-1$, and the optimal cost functional value at time step $k$ is denoted:

$$
L_{e}^{*}\left(x(k), u^{*}(\cdot \mid k)\right)=\sum_{j=k}^{k+N-1} l_{e}\left(x^{*}(j \mid k), u^{*}(j \mid k)\right),
$$


where $u^{*}(\cdot \mid k)$ is the optimal input sequence (trajectory) and $x^{*}(\cdot \mid k)$ is the corresponding state sequence starting at $x(k)$. Using the bound on the difference between the two consecutive cost functional values, the closed-loop average economic performance can be bounded:

$$
\frac{1}{T+1} \sum_{k=0}^{T} l_{e}\left(x(k), u^{*}(k \mid k)\right) \leq l_{e}\left(x_{s}^{*}, u_{s}^{*}\right)+\frac{L_{e}^{*}(0)-L_{e}^{*}(T)}{T+1}
$$

where $x(k)$ is the closed-loop state at time step $k, L_{e}^{*}(j)$, with abuse of notation, denotes the optimal cost functional value at time step $j$, and $T<\infty$ is the length of operation. From (14), the effect of the second term of the right-hand side dissipates with longer (but finite) operation. For infinite-time, the average economic performance is bounded by:

$$
\limsup _{T \rightarrow \infty} \frac{1}{T+1} \sum_{k=0}^{T} l_{e}\left(x(k), u^{*}(k \mid k)\right) \leq l_{e}\left(x_{s}^{*}, u_{s}^{*}\right),
$$

that is, the asymptotic average performance is no worse than that at the pair $\left(x_{s}^{*}, u_{s}^{*}\right)$ (Angeli et al. (2012)).

Remark 4. If the dynamic constraints (3) take the form of average constraints, Angeli et al. (2012) and Müller et al. (2014b) provide methodologies for EMPC with an equality terminal constraint to ensure that the average constraint is satisfied asymptotically and over finite-time operating horizons, respectively.

\subsection{EMPC with a Terminal Region Constraint}

EMPC with the equality terminal constraint (8) requires that the initial state be sufficiently close to the steady-state such that it is possible to reach the steadystate in $N$ sampling times. This type of constraint can limit the feasible region 
(Amrit et al. (2011)). Numerically computing a solution that satisfies such a constraint exactly may also be challenging. Therefore, terminal region constraints may be employed in EMPC.

One such method is a terminal region constraint designed via an auxiliary local control law. The terminal region is designed to be forward invariant for the nonlinear system under the local control law. The local control law can, for instance, be designed on the basis of the linearization of the system around the optimal steadystate. The terminal region is denoted as $\mathbb{X}_{f}$ and the terminal constraint imposed in the EMPC problem is

$$
\tilde{x}\left(t_{k+N}\right) \in \mathbb{X}_{f}
$$

In general, for closed-loop stability and performance, the terminal cost is such that $V_{f}(\cdot) \not \equiv 0$.

In Amrit et al. (2011), a procedure to design a local control law, a terminal region constraint, and a terminal cost for EMPC satisfying the assumption below was proposed:

Assumption 1. There exist a compact terminal region $\mathbb{X}_{f} \subset \mathbb{R}^{n}$, containing the point $x_{s}^{*}$ in its interior, and control law $h_{L}: \mathbb{X}_{f} \rightarrow \mathbb{U}$, such that (for the discretetime system of (9)):

$$
V_{f}\left(f_{d}\left(x, h_{L}(x)\right)\right) \leq V_{f}(x)-l_{e}\left(x, h_{L}(x)\right)+l_{e}\left(x_{s}^{*}, u_{s}^{*}\right)
$$

for all $x \in \mathbb{X}_{f}$. 


\subsubsection{Feasibility.}

For nominal operation, if the EMPC with a terminal region is initially feasible, the EMPC will be recursively feasible. This can be shown by using similar recursive arguments as those used in showing the feasibility of the EMPC with the equality terminal constraint. If $u^{*}\left(t \mid t_{k-1}\right)$ for $t \in\left[t_{k-1}, t_{k+N-1}\right)$ is the optimal input trajectory at $t_{k-1}$, then at $t_{k}$, a feasible solution is $u(t)=u^{*}\left(t \mid t_{k-1}\right)$ for $t \in$ $\left[t_{k}, t_{k+N-1}\right)$ and $u(t)=h_{L}\left(\tilde{x}\left(t_{k+N-1}\right)\right)$ for $t \in\left[t_{k+N-1}, t_{k+N}\right)$ where $\tilde{x}\left(t_{k+N-1}\right)$ is the predicted state at $t_{k+N-1}$. For recursive feasibility when $w(\cdot) \not \equiv 0$, one EMPC methodology designed with a terminal region constraint was presented in Bayer et al. (2014).

\subsubsection{Closed-loop Stability.}

The closed-loop stability properties of EMPC with a terminal constraint designed to satisfy Assumption 1 are similar to those of EMPC with an equality terminal constraint. For nominal operation, the closed-loop state trajectory will stay in the feasible region. If the system (9) is strictly dissipative with supply rate (11), the steady-state is asymptotically stable under EMPC with a terminal region constraint (Amrit et al. (2011); see, also, Alessandretti et al. (2014) which extends these results to continuous-time systems).

\subsubsection{Closed-loop Performance.}

If the local control law, terminal cost, and terminal region are designed such that Assumption 1 is satisfied, the bound on asymptotic average performance (15) holds (Amrit et al. (2011)). For finite-time, a similar bound as the bound (14) may be derived for the closed-loop system under EMPC with a terminal cost and 
terminal region.

\subsection{Lyapunov-based EMPC}

The feasible region of EMPC with a terminal region constraint, while larger than the feasible region of EMPC with an equality terminal constraint, depends on the prediction horizon length. As previously pointed out, the feasible region of both aforementioned EMPC formulations is difficult to characterize. As an alternative to overcome these challenges, one can consider designing an explicit nonlinear control law for the system (1) (as opposed to a local stabilizing controller) and constructing a Lyapunov function for the resulting closed-loop system consisting of the system (1) under the explicit nonlinear control law. With the control law and Lyapunov function, a region constraint may be designed to be imposed within EMPC. Because the control law and Lyapunov function are derived for the closed-loop system, the resulting region constraint also provides an estimate of the region of attraction of the nonlinear system (1) under the Lyapunov-based controller. Here, continuous-time systems are considered, and the effect of sampling on closed-loop stability is explicitly considered. Also, bounded disturbances and other forms of uncertainty (e.g., discretization error) are taken into account.

The existence of a feedback controller $h: \mathbb{R}^{n} \rightarrow \mathbb{U}$ is assumed which renders the origin of the nominal closed-loop system $\dot{x}=f(x, h(x), 0)$ asymptotically stable (e.g., Khalil (2002)) in the sense that there exists a continuously differentiable Lyapunov function $V: \mathbb{R}^{n} \rightarrow \mathbb{R}$ that satisfies the following inequalities:

$$
\alpha_{1}(|x|) \leq V(x) \leq \alpha_{2}(|x|)
$$




$$
\frac{\partial V(x)}{\partial x} f(x, h(x), 0) \leq-\alpha_{3}(|x|)
$$

for all $x \in D$ where $\alpha_{i}, i=1,2,3$ are class $\mathcal{K}$ functions and $D$ is an open neighborhood of the origin. A level set $\Omega_{\rho} \subseteq D$ of the Lyapunov function (i.e., $\Omega_{\rho}:=\left\{x \in \mathbb{R}^{n}: V(x) \leq \rho\right\}$ ) within which all state constraints are met $\left(\Omega_{\rho} \subseteq \mathbb{X}\right)$ and within which the time-derivative of $V$ is negative along the trajectories of the closed-loop system under the explicit controller $h$ is an estimate of the stability region of the closed-loop system. A number of works have addressed the development of control laws for various classes of nonlinear systems including those that ensure the satisfaction of input constraints and for which the region of attraction of the closed-loop system under the given control law can be explicitly characterized (see, for example, Lin and Sontag (1991); Sontag (1998); Kokotović and Arcak (2001); Christofides and El-Farra (2005) and the references therein for results in this direction). Practical stability of the origin of the closed-loop system results when the feedback controller is applied in a sample-and-hold fashion with a sufficiently small sampling period (see, for instance, Muñoz de la Peña and Christofides (2008) and the references therein for results and analysis of sampled-data systems). Moreover, for the resulting sampleddata system consisting of the continuous-time system (1) and the feedback controller applied in a sample-and-hold fashion, the region $\Omega_{\rho}$ is forward invariant and $V$ is a (practical) Lyapunov function (e.g., Muñoz de la Peña and Christofides $(2008))$.

Utilizing the stability region $\Omega_{\rho}$, the following EMPC formulation, which is 
the so-called Lyapunov-based EMPC (LEMPC) (Heidarinejad et al. (2012a)), has been proposed. LEMPC is a two-mode control strategy, and its problem formulation is given by the following OCP:

$$
\begin{array}{ll}
\min _{u(\cdot) \in S(\Delta)} & \int_{t_{k}}^{t_{k+N}} l_{e}(\tilde{x}(t), u(t)) d t \\
\text { s.t. } \quad & \dot{x}(t)=f(\tilde{x}(t), u(t), 0) \\
& \tilde{x}\left(t_{k}\right)=x\left(t_{k}\right) \\
u(t) \in \mathbb{U}, \forall t \in\left[t_{k}, t_{k+N}\right) \\
V(\tilde{x}(t)) \leq \rho_{e}, \forall t \in\left[t_{k}, t_{k+N}\right] \\
\quad \text { if } V\left(x\left(t_{k}\right)\right) \leq \rho_{e} \text { and } t_{k}<t_{s} \\
\frac{\partial V}{\partial x} f\left(x\left(t_{k}\right), u\left(t_{k}\right), 0\right) \leq \frac{\partial V}{\partial x} f\left(x\left(t_{k}\right), h\left(x\left(t_{k}\right)\right), 0\right) \\
\text { if } V\left(x\left(t_{k}\right)\right)>\rho_{e} \text { or } t_{k} \geq t_{s}
\end{array}
$$

where $t_{s}$ is the switching time of the controller, which is selected based on practical considerations for a given process, as will be discussed below. The LEMPC problem is similar to the general EMPC problem (5) with two Lyapunov-based constraints (19e)-(19f), which define the two modes of operation. Only one of these two constraints are imposed at any given sampling time. The constraint (19e), which defines mode 1 operation, constrains the predicted state trajectory to be contained in a subset of the stability region $\left(\Omega_{\rho_{e}}=\left\{x \in \mathbb{R}^{n}: V(x) \leq\right.\right.$ $\left.\rho_{e}\right\} \subset \Omega_{\rho}$ ). The constraint (19f), which defines mode 2 operation, is a contractive constraint imposed on the control action computed for the first sampling period of the horizon. Both constraints are explained below. 


\subsubsection{Feasibility.}

The LEMPC optimization problem is feasible for any initial state in $\Omega_{\rho}$. This follows because the sample-and-hold input trajectory obtained from the explicit controller is a feasible solution to the optimization problem for either mode of operation. For $x\left(t_{k}\right) \in \Omega_{\rho}, u(t)=\hat{u}(t)$ for $t \in\left[t_{k}, t_{k+N}\right)$ is a feasible input trajectory where $\hat{u}$ is the resulting input trajectory of the system:

$$
\begin{aligned}
& \dot{\hat{x}}(t)=f(\hat{x}(t), \hat{u}(t), 0), \hat{x}\left(t_{k}\right)=x\left(t_{k}\right) \\
& \hat{u}(t)=h\left(\hat{x}\left(t_{j}\right)\right), \text { for } t \in\left[t_{j}, t_{j+1}\right)
\end{aligned}
$$

for $j=k, k+1, \ldots, k+N-1$. For mode 1 operation, $\Omega_{\rho_{e}}$ is forward invariant for the nominal system under the sample-and-hold controller (assuming that $\Omega_{\rho_{e}}$ is an appropriately chosen subset of $\Omega_{\rho}$ ). Also, the input trajectory $\hat{u}$ (trivially) satisfies the contractive constraint (19f) making it a feasible solution under mode 2 operation. Recursive feasibility of the LEMPC follows if the closedloop state trajectory is maintained in $\Omega_{\rho}$, which is guaranteed by the dual-mode implementation.

\subsubsection{Closed-loop Stability.}

The motivation for the design of LEMPC is that forcing the state to converge to a small neighborhood of the steady-state and maintaining the state within this neighborhood thereafter (i.e., steady-state operation) may not be the best operation strategy from an economic perspective. For example, the best operating strategy may be some periodic operating policy. Moreover, the LEMPC design seeks to provide guarantees on recursive feasibility and closed-loop stability for operation 
affected by sufficiently small bounded disturbances or uncertainty. Thus, mode 1 operation allows for the LEMPC to enforce a bounded time-varying operating policy that optimizes the economics while maintaining the closed-loop state trajectory inside $\Omega_{\rho}$ in the presence of bounded disturbances or other sources of uncertainty. The set $\Omega_{\rho_{e}}$ is designed such that if the current state $x\left(t_{k}\right) \in \Omega_{\rho_{e}}$ and the predicted state at the next sampling time $\tilde{x}\left(t_{k+1}\right) \in \Omega_{\rho_{e}}$, then the actual state at the next sampling time, which may be forced away from $\Omega_{\rho_{e}}$ owing to a disturbance/uncertainty, will be in $\Omega_{\rho}$. For any state in $\Omega_{\rho} \backslash \Omega_{\rho_{e}}$ or if it is desirable to force convergence of the state to a neighborhood of the steady-state, the contractive Lyapunov-based constraint (19f) is used. The constraint guarantees that the Lyapunov function value will decrease at the next sampling time. Under mode 1 operation, the closed-loop state trajectory will remain bounded in $\Omega_{\rho}$, and under mode 2 operation, the state will converge to $\Omega_{\rho_{e}}$ in a finite number of sampling times for any $x\left(t_{k}\right) \in \Omega_{\rho} \backslash \Omega_{\rho_{e}}$. After $t_{s}$, the state will converge to a small compact forward invariant set containing the origin (Heidarinejad et al. (2012a)).

The two tuning parameters of LEMPC are the switching time $t_{s}$ and the set $\Omega_{\rho_{e}}$. If $t_{s}=0$, the LEMPC will always operate in mode 2. This may be desirable if steady-state operation is expected and/or is the best operating strategy. A strict dissipativity assumption with a supply rate that depends on the economic stage cost does not need to hold for this case to ensure convergence of the closed-loop state trajectory to a neighborhood of the optimal steady-state. If $t_{s} \rightarrow \infty$, the LEMPC may dictate a time-varying operating policy over the entire length of operation. An intermediate choice for the switching time $\left(t_{s} \in(0, \infty)\right)$ may be used 


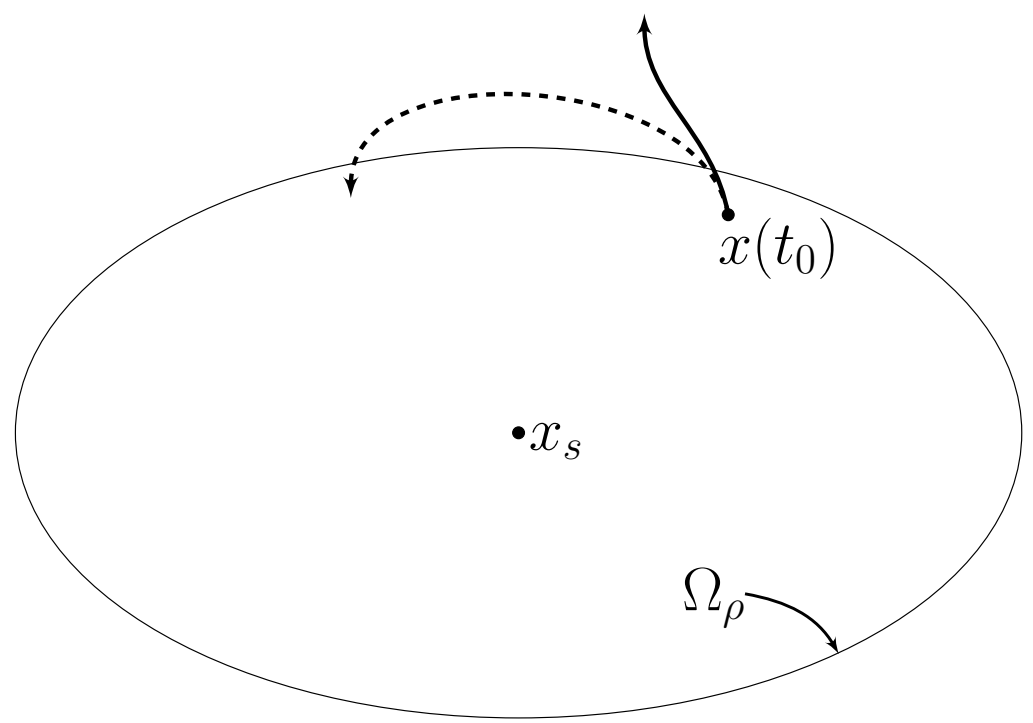

Figure 1: An illustration of the possible consequence of using the stability region $\Omega_{\rho}$ as a terminal region constraint. The dashed trajectory is the open-loop predicted state trajectory of the EMPC, while the solid trajectory is the closed-loop state trajectory.

to balance the trade-off between achieving better economic performance through time-varying operation and excessive control actuator wear required to enforce the time-varying operating policy. The other tuning parameter of LEMPC is $\rho_{e}$ which does not need to be chosen so that $\Omega_{\rho_{e}}$ is the largest subset of $\Omega_{\rho}$ such that the state at the next sampling time is guaranteed to be in $\Omega_{\rho}$ under mode 1 operation of the LEMPC. A larger set $\Omega_{\rho_{e}}$ may allow for better closed-loop economic performance. On the other hand, a smaller set $\Omega_{\rho_{e}}$ may allow for more robustness to uncertainty.

In contrast to EMPC with a terminal region constraint based on a local control law, the region constraint $(19 \mathrm{e})$ is imposed over the prediction horizon. For any state contained in $\Omega_{\rho}$, there is an a priori guarantee that there is a feasible control action that will lead to closed-loop stability in the sense of boundedness 
of the state inside $\Omega_{\rho}$ in the presence of bounded disturbances (i.e., there is a degree of robustness to uncertainty). To increase the feasible set under LEMPC, one may attempt to impose the region constraint (19e) as a terminal region constraint. While this may potentially increase the feasible region of the resulting EMPC, closed-loop stability (boundedness of the state in the feasible region) may only be guaranteed, in general, for nominal operation. In the presence of bounded disturbances, the closed-loop state trajectory may lead to infeasibility of the resulting EMPC problem or even closed-loop instability. The reason for this behavior is that the EMPC resulting from imposing the region constraint $19 \mathrm{e}$ as a terminal region constraint may allow for the closed-loop state to evolve out of $\Omega_{\rho}$. Once the closed-loop state is outside $\Omega_{\rho}$, robustness is not guaranteed meaning that a disturbance could force the state away from the predicted state trajectory that converges back to $\Omega_{\rho}$, and the result is that there is no feasible input trajectory that forces the state back to $\Omega_{\rho}$ (this is illustrated in Fig. 1).

\subsubsection{Closed-loop Performance.}

If one could design a terminal cost such that (17) is satisfied with the explicit controller, then a similar performance analysis as that of the EMPC with a terminal region constraint could be used to derive bounds on the closed-loop performance under LEMPC given that the closed-loop state trajectory is bounded in the compact set $\Omega_{\rho}$. Systematic methods of designing such a terminal cost that satisfies (17) as well as addressing key technical considerations remain open problems. However, in Liu et al. (2015), a terminal cost design methodology was proposed that takes advantage of an explicit controller using a different approach. 
One may also consider methods of constructing a terminal equality constraint that accounts for the closed-loop performance over a finite operating window to obtain closed-loop performance guarantees under LEMPC (Ellis and Christofides $(2014 \mathrm{~b}))$. For example, the input profile and open-loop predicted state trajectory of a process under an auxiliary stabilizing controller such as the explicit controller or a tracking MPC could be computed over an operating window. The terminal state of the computed state trajectory (i.e., the state at the end of the operating window with the auxiliary controller) could be incorporated in the LEMPC as an equality terminal constraint. The closed-loop economic performance under LEMPC can be proven to be at least as good as the closed-loop economic performance under the auxiliary stabilizing controller on both the finite-time and infinite-time intervals (Ellis and Christofides (2014b, 2016)). Another idea for constraining the LEMPC using an auxiliary control law is to compute the total control energy used by the auxiliary stabilizing controller and enforce that the LEMPC computes an input trajectory that uses no more control energy than the auxiliary controller input profile over the operating window (Heidarinejad et al. (2013)). This may be particularly important when the economic stage cost does not penalize the use of control energy.

\subsubsection{Satisfying State Constraints.}

To clarify imposing state constraints within LEMPC, one can extend the concepts from LMPC (e.g., Mhaskar et al. (2006)) for imposing state constraints in LEMPC. Specifically, define the set $\Phi_{u}$ as the set in state-space that includes all the states where $\dot{V}<0$ under the explicit controller. Then, if $\Phi_{u} \subseteq \mathbb{X}$, any initial 


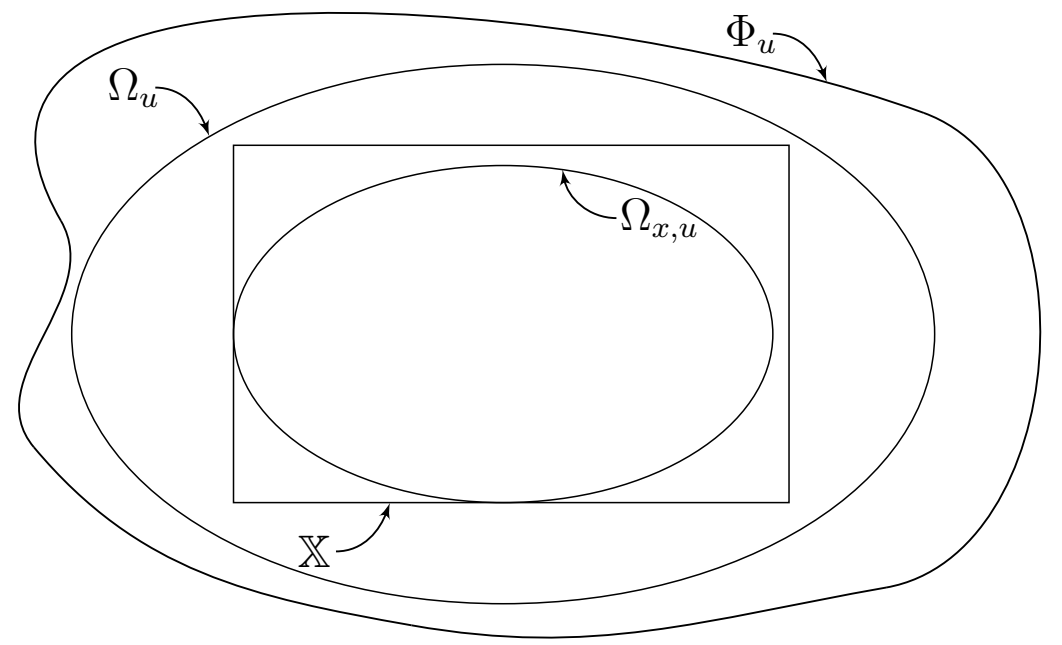

Figure 2: An illustration of the state-space sets discussed with respect to LEMPC satisfying state constraints. This illustration specifically considers the case when $\mathbb{X} \subset \Phi_{u}$.

state starting in the region $\mathbb{X} \backslash \Phi_{u}$ satisfies the state constraint. However, because the region $\mathbb{X} \backslash \Phi_{u}$ includes state-space points at which the time-derivative of the Lyapunov function may be positive, there may be initial conditions in that region for which the closed-loop system cannot be stabilized. Thus, in this case, the stability region used in the formulation of the LEMPC is $\Omega_{\rho}=\Omega_{x, u}=\left\{x \in \mathbb{R}^{n_{x}}\right.$ : $\left.V(x) \leq \rho_{x, u}\right\}$ where $\rho_{x, u}$ is chosen such that $\Omega_{x, u} \subseteq \Phi_{u}$.

Alternatively, if $\mathbb{X} \subset \Phi_{u}$, which is the case depicted in Fig. 2, then any initial state in $\Phi_{u} \backslash \mathbb{X}$ violates the state constraint from the onset. Also, for any initial state in the set $\mathbb{X}$, it is not possible, in general, to guarantee that the set $\mathbb{X}$ is forward invariant because there may exist a stabilizing state trajectory (i.e., a trajectory where $\dot{V}<0$ ) that goes outside of the set $\mathbb{X}$ before it enters back into the set to converge to the origin. When the state constraints are imposed as hard constraints, 
define the set $\Omega_{\rho}$ as $\Omega_{\rho}=\Omega_{x, u}=\left\{x \in \mathbb{R}^{n_{x}}: V(x) \leq \rho_{x, u}\right\}$ where $\rho_{x, u}$ is such that $\Omega_{x, u} \subseteq \mathbb{X}$. Since $\Phi_{u}$ cannot be computed in practice, the set $\Omega_{u}:=\{x \in$ $\left.\mathbb{R}^{n}: V(x) \leq \rho_{u}\right\}$ where $\rho_{u}$ is such that $\dot{V}<0$ for all $x \in \Omega_{u}$ under the explicit controller may be used. The set $\Omega_{u}$ accounts for the input constraints, but not necessarily the state constraints. An illustration of the set definitions is provided in Fig. 2. The following illustrative example provides a demonstration of the above methodology to satisfy state constraints.

\section{Example 1. Consider}

$$
\dot{x}(t)=x(t)+u(t)
$$

which has an open-loop unstable steady-state at the origin. First, consider that the system has the following constraints on the input and state: $u(t) \in \mathbb{U}=[-1,1]$ and $x(t) \in \mathbb{X}=[-2,2]$. For any initial state $x(0) \notin[-1,1]$, the state will diverge to positive/negative infinity (i.e., some initial states that satisfy the state constraint may result in a closed-loop trajectory that does not continue satisfying the state constraints over the length of operation). Following the approach detailed above, the nonlinear control law $u=-\operatorname{sat}(K x)$ where $\operatorname{sat}(\cdot)$ is the saturation function and $K>1$ is a tuning parameter renders the origin of (20) exponentially stable while satisfying the input constraints. The quadratic function:

$$
V(x)=x^{2}
$$

is a Lyapunov function for the closed-loop system. Moreover, the region where $\dot{V}<0$ while accounting for the input constraints is $\Omega_{u}=\left\{x \in \mathbb{R}: V(x) \leq \rho_{u}\right\}$ where $\rho_{u}$ is chosen such that $\rho_{u}<1$. Since $\Omega_{u} \subset \mathbb{X}, \Omega_{\rho}$ can be taken to be $\Omega_{u}$. 
If, instead, $\mathbb{X}=[-0.9,0.9]$, then $\mathbb{X} \subset \Omega_{u}$, and $\Omega_{\rho}$ can be taken to be $\{x \in \mathbb{R}:$ $V(x) \leq 0.81\}$ (i.e., $\Omega_{\rho}=\mathbb{X}$ ). In either situation, one can verify that for any initial state in $\Omega_{\rho}$, the closed-loop state trajectory will remain bounded in $\Omega_{\rho}$ and converge exponentially to the origin without violating the state constraints. If one were to apply LEMPC to the system designing the Lyapunov-based constraints with the control law, the LEMPC would inherit these properties as well.

\subsubsection{Real-time Implementation.}

As a by-product of designing the explicit nonlinear control law, it may be used as an auxiliary or back-up controller. For instance, a real-time implementation strategy for LEMPC, referred to as real-time LEMPC, was proposed in Ellis and Christofides (2015b) to account for possibly unknown and time-varying computational delay. The strategy uses triggered evaluations of the LEMPC to only recompute a solution when stability conditions dictate a new input trajectory must be computed. If the precomputed control action satisfies the stability conditions, the control action is applied to the closed-loop system. If not, the explicit control law, which has negligible computation time, is used to compute the control action for the system at the current sampling time.

\subsubsection{Input Rate of Change Constraints}

Mode 1 operation of LEMPC may cause excessive actuator wear if the control actions calculated by the LEMPC regularly change significantly between two sampling periods. One method for preventing the excessive wear without exiting mode 1 operation or restricting the manipulated input $u(t)$ to a subset of $\mathbb{U}$, which would likely reduce the economic performance of the LEMPC strategy compared 
to that which could be obtained, is by adding input rate of change constraints to the LEMPC. To reduce actuator wear, it is desirable to limit the difference between $u_{i}^{*}\left(t_{k} \mid t_{k}\right)$ and $u_{i}^{*}\left(t_{k-1} \mid t_{k-1}\right)$ for $i=1, \ldots, m$ to be within a desired range $\epsilon_{\text {desired }}$ as follows:

$$
\left|u_{i}^{*}\left(t_{k} \mid t_{k}\right)-u_{i}^{*}\left(t_{k-1} \mid t_{k-1}\right)\right| \leq \epsilon_{\text {desired }}, i=1, \ldots, m
$$

However, if this constraint is incorporated directly in the LEMPC of Eq. 19, the input trajectory $\hat{u}(t)$ that is feasible for all of the other LEMPC constraints cannot be proven to meet the constraint in Eq. 22. However, $\hat{u}(t)$ does satisfy the following constraint which can be added to the LEMPC instead:

$$
\left|u_{i}\left(t_{k}\right)-h_{i}\left(x\left(t_{k}\right)\right)\right| \leq \epsilon, i=1, \ldots, m
$$

The constraint in Eq. 23 is proven in Durand et al. (2015) to cause the desired input rate of change of Eq. 22 to be met for an appropriately chosen $\epsilon$ and a sufficiently small $\Delta$ when the component functions of the explicit controller $h$ are Lipschitz continuous, which is not practically restrictive.

The input rate of change constraints in Eqs. 22 and 23 are enforced for the first sampling period of the prediction horizon, meaning that they constrain only the control action that will be implemented on the process due to the receding horizon implementation of LEMPC. However, the rate of change constraint in Eq. 23 could be enforced at additional sampling periods in the prediction horizon, including throughout the entire prediction horizon, to satisfy the desired bound of Eq. 22 for each sampling period in the prediction horizon. Specifically, if the 
following constraints are added to the LEMPC:

$$
\begin{aligned}
& \left|u_{i}\left(t_{k}\right)-h_{i}\left(x\left(t_{k}\right)\right)\right| \leq \epsilon, i=1, \ldots, m \\
& \left|u_{i}\left(t_{j}\right)-h_{i}\left(\tilde{x}\left(t_{j}\right)\right)\right| \leq \epsilon, i=1, \ldots, m, j=k+1, \ldots, k+N-1
\end{aligned}
$$

then for an appropriate $\epsilon$ and $\Delta$, the following bounds are satisfied:

$$
\begin{aligned}
& \left|u_{i}^{*}\left(t_{k} \mid t_{k}\right)-u_{i}^{*}\left(t_{k-1} \mid t_{k-1}\right)\right| \leq \epsilon_{\text {desired }}, i=1, \ldots, m \\
& \left|u_{i}^{*}\left(t_{j} \mid t_{k}\right)-u_{i}^{*}\left(t_{j-1} \mid t_{k}\right)\right| \leq \epsilon_{\text {desired }} \\
& \quad \quad \quad \\
& \quad i=1, \ldots, m, j=k+1, \ldots, k+N-1
\end{aligned}
$$

The number of sampling periods over which the constraint in Eq. 23 is enforced (i.e., the choice to use Eq. 23 or 24 in Eq. 19) is an important consideration for the performance and constraint satisfaction of the LEMPC, especially if dynamic constraints like those in Eq. 5e are also used in the LEMPC. In general, LEMPC incorporating input rate of change constraints can be expected to perform more optimally throughout an operating period if it is aware of all constraints that will be enforced upon it throughout the operating period (i.e., the constraints in Eq. 24 are likely to provide the most economically optimal input trajectories). If, for example, the input rate of change constraint is only enforced for the first sampling period of the prediction horizon, the LEMPC may predict a trajectory with wide variations in the input after the first sampling period to maximize process economics, while choosing an input for the first sampling period with less economic benefit than might be obtained if it predicted that future trajectories would be similarly constrained. This would be especially important to consider if the 
LEMPC incorporated a dynamic constraint, because then the expected values of the inputs in the later sampling periods of the prediction horizon would affect the value chosen in the first sampling period. In addition, the ability of the process to meet all constraints is also impacted by the number of sampling periods over which the input rate of change constraints are enforced, particularly if dynamic constraints are used that require the states and inputs to meet certain metrics in a given timeframe, which implies that good forecasting of the process and input trajectories would be required in each sampling period to ensure that the control actions in each sampling period are contributing to this goal.

The enforcement of the input rate of change constraints in the LEMPC of Eq. 19 would be likely to reduce the profit below that which could be attained without the input rate of change constraints because they restrict the allowable control actions. However, there are many cases in which the LEMPC with input rate of change constraints will still significantly outperform steady-state operation (Durand et al. (2015)). In addition, economics-based cost functions do not typically reflect profit loss that may occur due to actuator wear and tear, so though an initial analysis of LEMPC with input rate of change constraints may suggest that the input rate of change constraints lead to a loss in profit compared to the case that input rate of change constraints are not used, the use of the input rate of change constraints to reduce actuator wear has the potential to improve control loop performance overall by reducing the likelihood that issues associated with the actuation element may occur. If the reduction in profit with the addition of such constraints remains a concern, however, and the constraints are only added 
to achieve more desirable trends in the inputs from the LEMPC instead of because of hard constraints on the processing equipment capabilities, one could consider formulating the input rate of change constraints as soft constraints or as constraints that are only enforced if the process economics are better than a certain threshold.

Because the input rate of change constraints in Eqs. 23 and 24 are designed so that $\hat{u}$ is a feasible solution, feasibility of the LEMPC of Eq. 19 with the input rate of change constraints in Eqs. 23 and 24 is ensured. In addition, the input rate of change constraints do not affect the closed-loop stability properties of the LEMPC, so it remains stable in the sense of maintaining boundedness of the closed-loop states in the presence of disturbances after the input rate of change constraints are added.

Remark 5. The three EMPC formulations with explicit stability constraints presented each have specific advantages and disadvantages. Assuming that feasibility is not an issue, EMPC with a terminal equality constraint is perhaps the easiest to implement because it does not require the derivation of a region constraint. On the other hand, the LEMPC design provides guaranteed closed-loop stability in the sense that the closed-loop state trajectory will be bounded in a well-defined state-space region and also, does not require a dissipativity assumption to be satisfied if steady-state operation is desired. However, the design of LEMPC requires the availability of an explicit stabilizing controller and a Lyapunov function for the nonlinear system.

Remark 6. The EMPC formulations may result in different open-loop predicted 


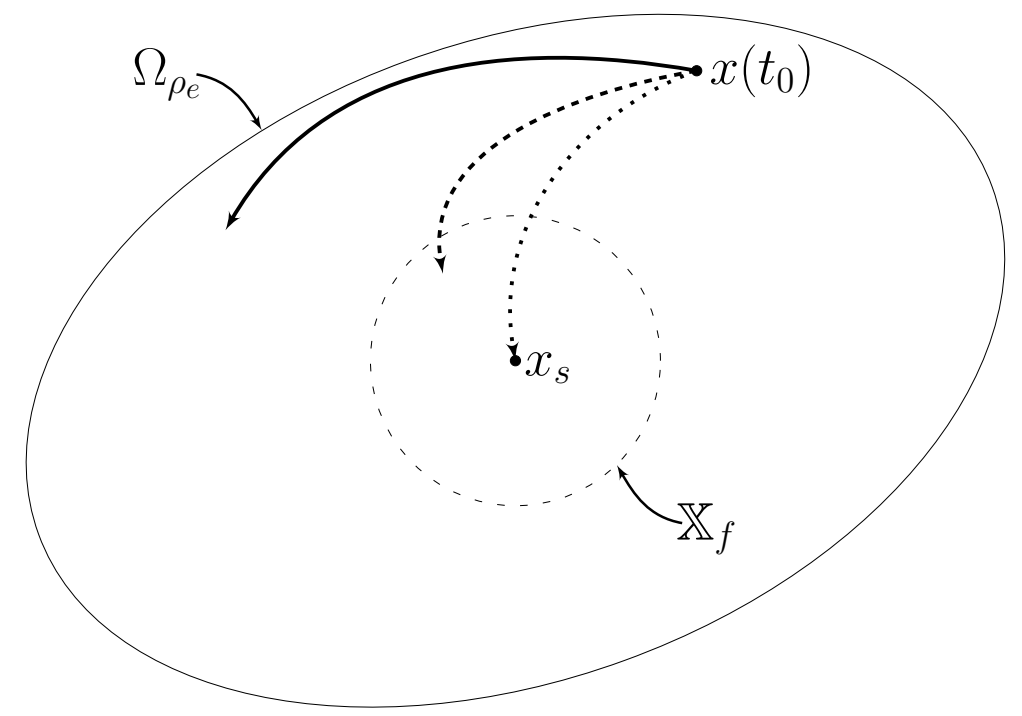

Figure 3: An illustration of possible open-loop predicted trajectories under EMPC formulated with a terminal constraint (dotted), under EMPC formulated with a terminal region constraint (dashed), and under LEMPC (solid).

state trajectories which are illustrated in Fig. 3, Nonetheless, if the prediction horizon is sufficiently long, the closed-loop behavior of the system under the various EMPC formulations would (intuitively) be expected to be similar because for a long prediction horizon, the EMPC solution starts to closely approximate the infinite horizon solution and the effect on the closed-loop behavior of the terminal conditions of the open-loop predicted trajectory is less significant than the corresponding effect for shorter prediction horizons.

\section{Performance Improvement Through Time-varying Operation}

A well-known chemical engineering example that demonstrates performance improvement through time-varying operation is examined to consider the role of 
Table 1: Process parameters of the CSTR.

\begin{tabular}{cc|cc}
\hline$k_{0}$ & $8.46 \times 10^{6}$ & $A_{1}$ & $1.69 \times 10^{6}$ \\
$x_{20}$ & 0.050 & $A_{2}$ & $1.41 \times 10^{4}$ \\
\hline
\end{tabular}

economic-oriented constraints on the optimal operating strategy and to motivate future research on time-varying operation under EMPC. In this illustration, stability is not considered. The necessity for feedback control is discussed at the end of the illustration.

Consider the system described by the following dynamic equations given in dimensionless form:

$$
\begin{aligned}
& \dot{x}_{1}=-x_{1}-A_{1} e^{-1 / x_{2}} x_{1}^{2}+u \\
& \dot{x}_{2}=-x_{2}+A_{2} e^{-1 / x_{2}} x_{1}^{2}+x_{20}
\end{aligned}
$$

where $x_{1}$ is the dimensionless reactant concentration, $x_{2}$ is the dimensionless temperature, and $A_{1}, A_{2}$ and $x_{20}$ are constant parameters. The values of the parameters are given in Tbl. 1. The input is bounded: $u \in\left[u_{\min }, u_{\max }\right]=[0.5,7.5]$. The system (26) describes a non-isothermal CSTR where a second-order reaction occurs and the inlet concentration of the reactant material is the manipulated input (Fig. (4). The economic stage cost is

$$
l_{e}(x, u)=k_{0} e^{-1 / x_{2}} x_{1}^{2}
$$

which is the production rate of the desired product ( $k_{0}$ is a parameter). The system has an input average constraint (dynamic constraint) given by:

$$
\frac{1}{t_{f}} \int_{0}^{t_{f}} u(t) d t=u_{\text {avg }}=4.0
$$




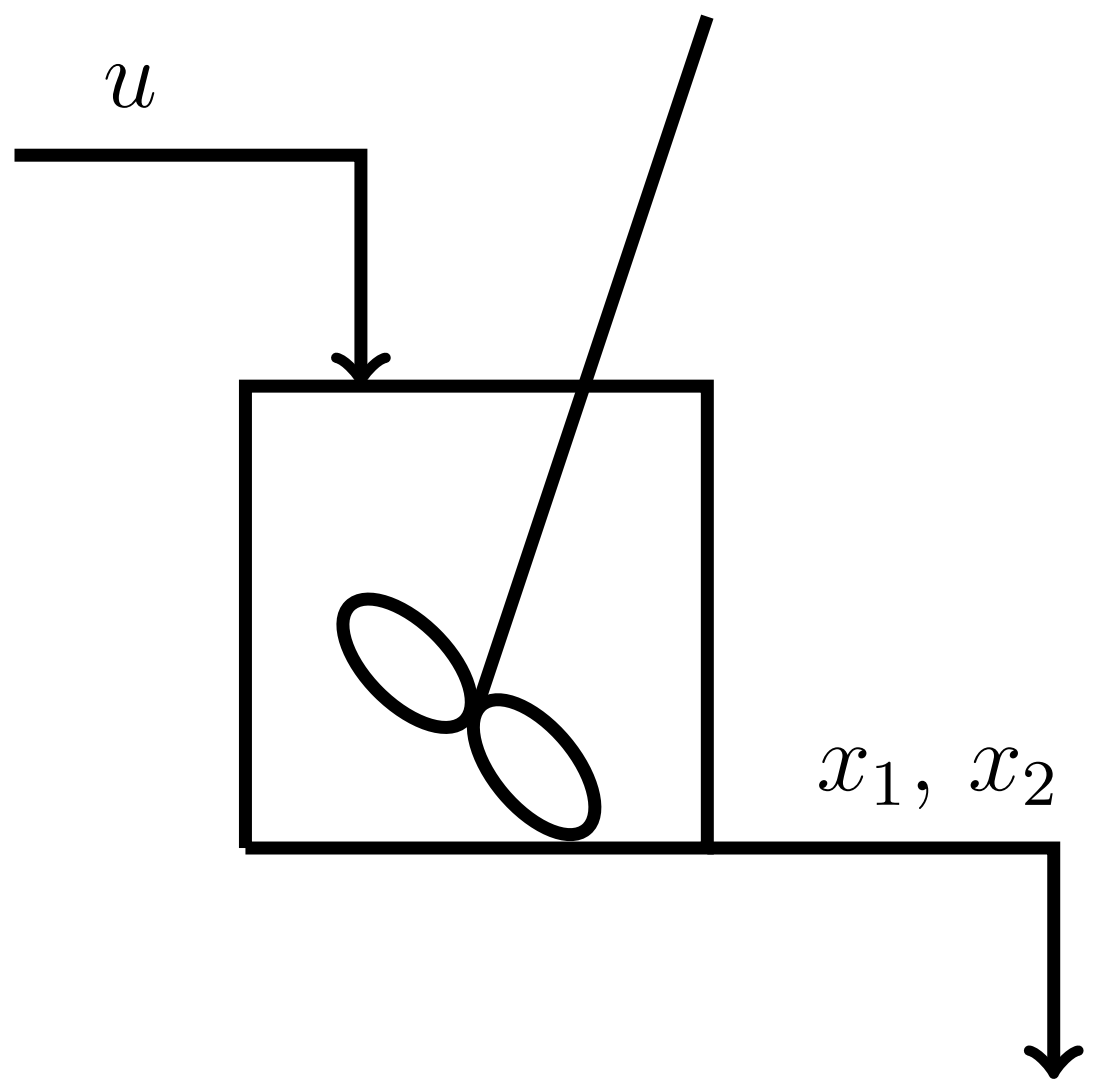

Figure 4: Depiction of CSTR considered in the chemical process example.

where $t_{f}$ is the length of operation. The practical motivation of the average constraint $(28)$ is that the average amount of material that may be distributed to the reactor over time is fixed (i.e., the constraint is economically motivated). The CSTR has an optimal steady-state $x_{s}^{T}=\left[\begin{array}{ll}1.182 & 0.073\end{array}\right]$ which corresponds to the steady-state input that satisfies the average input constraint $\left(u_{s}=u_{\text {avg }}\right)$ with a production rate of 14.03 .

An analysis is completed to determine if the economic performance (i.e., the average production rate of the product) can be improved by using a time-varying 
operating strategy compared to operating at the optimal steady-state. An auxiliary state is defined for the average constraint:

$$
x_{3}(t):=\frac{1}{t_{f}} \int_{0}^{t}\left(u(t)-u_{\text {avg }}\right) d t
$$

which has dynamics:

$$
\dot{x}_{3}(t)=\frac{1}{t_{f}}\left(u(t)-u_{\text {avg }}\right) .
$$

The non-isothermal CSTR with the objective function (27) is a member of a special class of nonlinear systems:

$$
\dot{x}=\bar{f}(x)+B u
$$

where $B \in \mathbb{R}^{n} \times \mathbb{R}^{m}$ is a constant matrix and $\bar{f}: \mathbb{R}^{n} \rightarrow \mathbb{R}^{n}$ is a differentiable vector function. Additionally, the stage cost only depends on the states:

$$
l_{e}(x, u)=\bar{l}_{e}(x)
$$

where $\bar{l}_{e}: \mathbb{R}^{n} \rightarrow \mathbb{R}$ is a differentiable function. The Hamiltonian function of the system (31) and cost (32) is

$$
H(x, u, \lambda)=\bar{l}_{e}(x)+\lambda^{T} \bar{f}(x)+\lambda^{T} B u
$$

where $\lambda$ is the adjoint variable vector that satisfies

$$
\dot{\lambda}(t)=-H_{x}(x(t), u(t), \lambda(t))
$$

where $H_{x}$ denotes the partial derivative of $H$ with respect to $x$. From Pontryagin's maximum principle (Pontryagin et al. (1961)), a necessary condition can be 


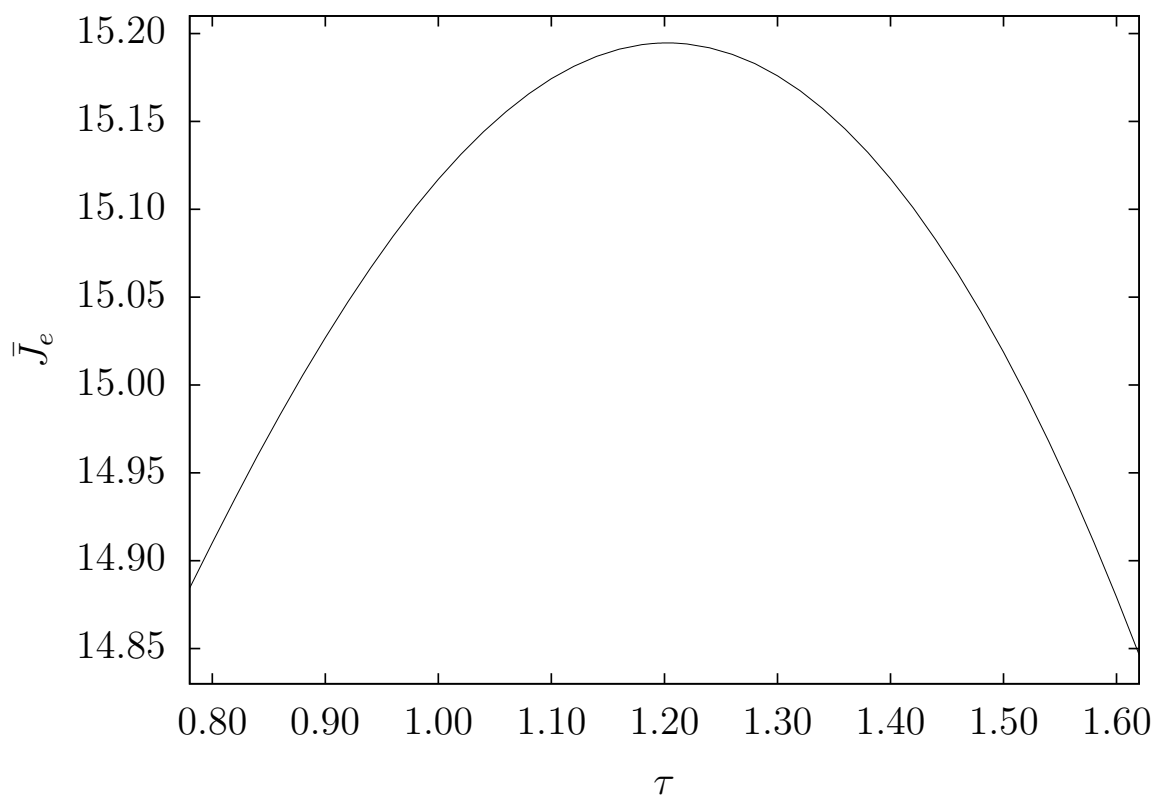

Figure 5: Average economic performance $\bar{J}_{e}$ as a function of the period length $\tau$.

derived for the optimal control (i.e., the control that maximizes the Hamiltonian):

$$
u_{i}^{*}(t)= \begin{cases}u_{i, \max } & \text { if } b_{i}^{T} \lambda(t)>0 \\ u_{i, \min } & \text { if } b_{i}^{T} \lambda(t)<0\end{cases}
$$

where $b_{i}$ is the $i$-th column of $B$. For this class of systems and stage costs, if some time-varying operating policy is the optimal operating strategy, then the operating policy is a bang-bang input policy (35).

Although the analysis above significantly reduces the space of input trajectories that one must consider to find the optimal control, it still yields an infinite space of input trajectories. Thus, consider the following periodic bang-bang input trajectory over one period:

$$
u(t)= \begin{cases}u_{\max } & \text { if } t<\tau / 2 \\ u_{\min } & \text { else }\end{cases}
$$



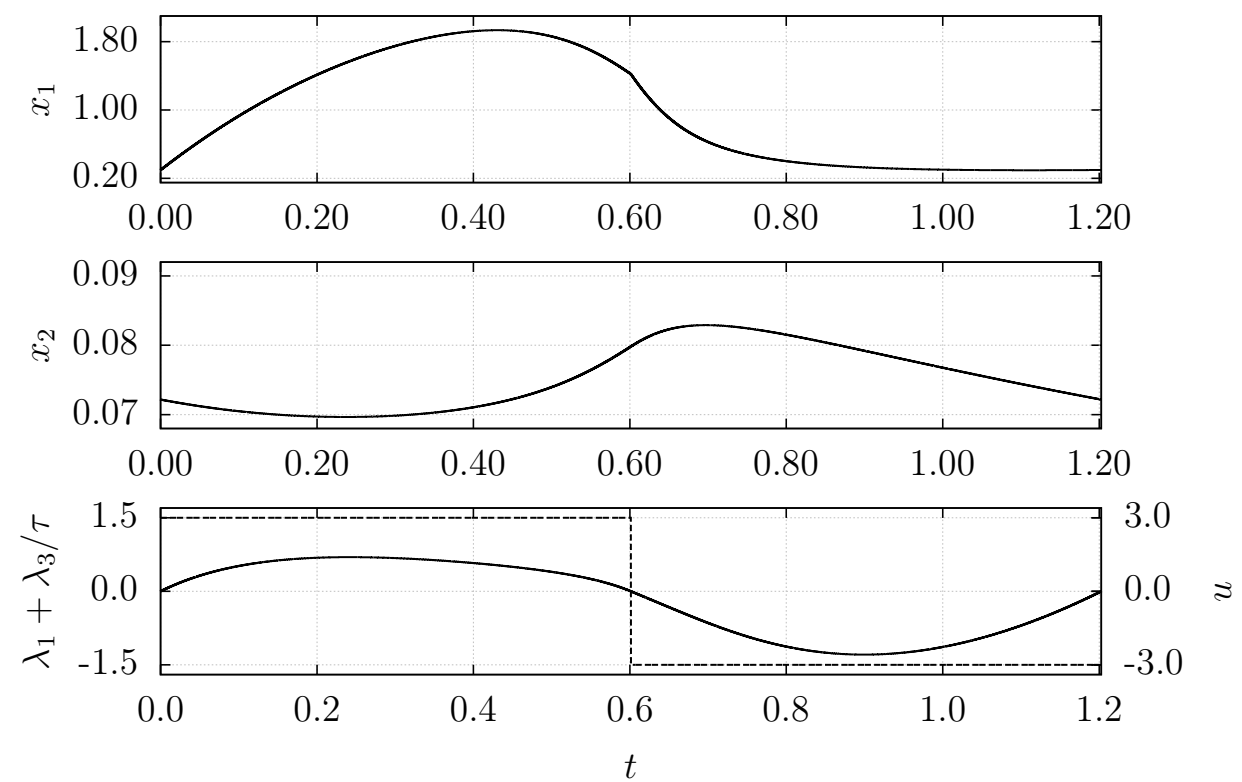

Figure 6: State, input, and $\lambda_{1}+\lambda_{3} / \tau$ trajectories of the CSTR under the bang-bang input policy with period $\tau=1.20$.

where $\tau$ is the period and $t \in[0, \tau)$. The input trajectory of (36) satisfies the average constraint of (28) over each period. For the system (26) with the input trajectory (36), there exists a periodic state trajectory for some $\tau>0$ (i.e., it has the property $x(t)=x(t+\tau)$ for all $t$ ).

Indeed, the periodic solution of the system (26) with the input (36) achieves better economic performance compared to the economic performance at steadystate for some $\tau$. Moreover, the economic performance depends on the period which is shown in Fig. 5. Over the range of periods considered (0.5 to 2.4), the period $\tau=1.20$ yields the best performance (Fig. [5). The periodic solution with the input period of $\tau=1.20$ has an average cost of $\bar{J}_{e}=15.20$ which is 8.30 percent better than the performance at the optimal steady-state. Periods greater 
than 1.96 achieve worse performance compared to that at steady-state. The state, input, and $B^{T} \lambda=b_{1}^{T} \lambda=\lambda_{1}+\lambda_{3} / \tau$ trajectories are given in Fig. 6 over one period. From Fig. 6, the input trajectory satisfies the necessary condition (35). From these results, time-varying operation is better than steady-state operation from an economical point of view for this example. If the average constraint (28) was not imposed, the optimal operating strategy would be steady-state operation at the steady-state corresponding to the input $u_{\max }$. The average constraint plays a crucial role for this particular example.

As pointed out, the above analysis only considers economic performance. If the periodic solution depicted in Fig. 6 is indeed optimal or some other bang-bang policy is the best operating strategy, feedback control is needed to force the system state from an initial state to the optimal time-varying solution. Moreover, the control problem becomes more complex when one considers disturbances, plantmodel mismatch and other forms of uncertainty, implementability of the computed input trajectory (i.e., bang-bang control may not be implementable in practice), and time-varying economic objectives and constraints. For this example, the application of EMPC has been studied and results in performance improvement over steady-state while addressing stability and robustness (Ellis et al. (2014)). The example further motivates the inquiry and theoretical developments in the context of EMPC systems that dictate time-varying operating policies. 


\section{Acknowledgements}

Financial support from the National Science Foundation and the Department of Energy is gratefully acknowledged.

\section{Vitae}

Matthew Ellis received his Ph.D. in Chemical Engineering from the University of California, Los Angeles, in 2015 and joined Johnson Controls. He is currently a Principal Engineer. His research interests are in economic model predictive control and optimization.

Helen Durand is currently a Ph.D. candidate in Chemical Engineering at the University of California, Los Angeles, with research focused on process control. She previously worked as an engineer in Materials \& Processes Engineering at Aerojet Rocketdyne, and has received both her B.S. and M.S. degrees from the University of California, Los Angeles.

Panagiotis D. Christofides is a Professor of Chemical and Biomolecular Engineering and of Electrical Engineering at the University of California, Los Angeles. His research interests are in control theory and process control. He is a Fellow of AAAS, IEEE and IFAC. 


\section{References}

Alessandretti, A., Aguiar, A., Jones, C., 2014. An economic model predictive control scheme with terminal penalty for continuous-time systems, in: Proceedings of the 53rd IEEE Conference on Decision and Control, Los Angeles, CA. pp. $2728-2733$.

Amrit, R., Rawlings, J.B., Angeli, D., 2011. Economic optimization using model predictive control with a terminal cost. Annual Reviews in Control 35, 178186.

Angeli, D., Amrit, R., Rawlings, J.B., 2012. On average performance and stability of economic model predictive control. IEEE Transactions on Automatic Control 57, 1615-1626.

Bayer, F.A., Müller, M.A., Allgöwer, F., 2014. Tube-based robust economic model predictive control. Journal of Process Control 24, 1237-1246.

Biegler, L.T., Yang, X., Fischer, G.A.G., 2015. Advances in sensitivity-based nonlinear model predictive control and dynamic real-time optimization. Journal of Process Control 30, 104-116.

Christofides, P.D., El-Farra, N.H., 2005. Control of Nonlinear and Hybrid Process Systems: Designs for Uncertainty, Constraints and Time-Delays. SpringerVerlag, Berlin, Germany.

Diehl, M., Amrit, R., Rawlings, J.B., 2011. A Lyapunov function for economic 
optimizing model predictive control. IEEE Transactions on Automatic Control $56,703-707$.

Durand, H., Ellis, M., Christofides, P.D., 2015. Economic model predictive control designs for input rate-of-change constraint handling and guaranteed economic performance. Computers \& Chemical Engineering, submitted.

Ellis, M., Christofides, P.D., 2014a. Economic model predictive control with timevarying objective function for nonlinear process systems. AIChE Journal 60, 507-519.

Ellis, M., Christofides, P.D., 2014b. On finite-time and infinite-time cost improvement of economic model predictive control for nonlinear systems. Automatica $50,2561-2569$.

Ellis, M., Christofides, P.D., 2015a. Economic model predictive control: Elucidation of the role of constraints, in: Proceedings of 5th IFAC Conference on Nonlinear Model Predictive Control, Seville, Spain. pp. 47-56.

Ellis, M., Christofides, P.D., 2015b. Real-time economic model predictive control of nonlinear process systems. AIChE Journal 61, 555-571.

Ellis, M., Christofides, P.D., 2016. On closed-loop average economic performance under Lyapunov-based economic model predictive control, in: Proceedings of the American Control Conference, Boston, MA. p. in press.

Ellis, M., Durand, H., Christofides, P.D., 2014. A tutorial review of economic model predictive control methods. Journal of Process Control 24, 1156-1178. 
Engell, S., 2007. Feedback control for optimal process operation. Journal of Process Control 17, 203-219.

Fagiano, L., Teel, A.R., 2013. Generalized terminal state constraint for model predictive control. Automatica 49, 2622-2631.

Faulwasser, T., Korda, M., Jones, C.N., Bonvin, D., 2014. Turnpike and dissipativity properties in dynamic real-time optimization and economic MPC, in: Proceedings of the 53rd IEEE Annual Conference on Decision and Control, Los Angeles, CA. pp. 2734-2739.

Ferramosca, A., Rawlings, J.B., Limon, D., Camacho, E.F., 2010. Economic MPC for a changing economic criterion, in: Proceedings of the 49th IEEE Conference on Decision and Control, Atlanta, GA. pp. 6131-6136.

Grosso, J.M., Ocampo-Martinez, C., Puig, V., Limon, D., Pereira, M., 2014. Economic MPC for the management of drinking water networks, in: Proceedings of the 2014 European Control Conference, Strasbourg, France. pp. 790-795.

Grüne, L., 2013. Economic receding horizon control without terminal constraints. Automatica 49, 725-734.

Grüne, L., Stieler, M., 2014. Asymptotic stability and transient optimality of economic MPC without terminal conditions. Journal of Process Control 24, 1187-1196.

Heidarinejad, M., Liu, J., Christofides, P.D., 2012a. Economic model predictive 
control of nonlinear process systems using Lyapunov techniques. AIChE Journal $58,855-870$.

Heidarinejad, M., Liu, J., Christofides, P.D., 2012b. State-estimation-based economic model predictive control of nonlinear systems. Systems \& Control Letters $61,926-935$.

Heidarinejad, M., Liu, J., Christofides, P.D., 2013. Algorithms for improved fixedtime performance of Lyapunov-based economic model predictive control of nonlinear systems. Journal of Process Control 23, 404-414.

Helbig, A., Abel, O., Marquardt, W., 2000. Structural concepts for optimization based control of transient processes, in: Allgöwer, F., Zheng, A. (Eds.), Nonlinear Model Predictive Control. Birkhäuser Basel. volume 26 of Progress in Systems and Control Theory, pp. 295-311.

Huang, R., Biegler, L.T., Harinath, E., 2012. Robust stability of economically oriented infinite horizon NMPC that include cyclic processes. Journal of Process Control 22, 51-59.

Huang, R., Harinath, E., Biegler, L.T., 2011. Lyapunov stability of economically oriented NMPC for cyclic processes. Journal of Process Control 21, 501-509.

Kadam, J.V., Marquardt, W., 2007. Integration of economical optimization and control for intentionally transient process operation, in: Findeisen, R., Allgöwer, F., Biegler, L.T. (Eds.), Assessment and Future Directions of Non- 
linear Model Predictive Control. Springer Berlin Heidelberg. volume 358 of Lecture Notes in Control and Information Sciences, pp. 419-434.

Khalil, H.K., 2002. Nonlinear Systems. Third ed., Prentice Hall, Upper Saddle River, NJ.

Kokotović, P., Arcak, M., 2001. Constructive nonlinear control: A historical perspective. Automatica 37, 637-662.

Limon, D., Pereira, M., Muñoz de la Peña, D., Alamo, T., Grosso, J.M., 2014. Single-layer economic model predictive control for periodic operation. Journal of Process Control 24, 1207-1224.

Lin, Y., Sontag, E.D., 1991. A universal formula for stabilization with bounded controls. Systems \& Control Letters 16, 393-397.

Liu, S., Zhang, J., Liu, J., 2015. Economic MPC with terminal cost and application to an oilsand primary separation vessel. Chemical Engineering Science $136,27-37$.

Mayne, D.Q., Rawlings, J.B., Rao, C.V., Scokaert, P.O.M., 2000. Constrained model predictive control: Stability and optimality. Automatica 36, 789-814.

Mhaskar, P., El-Farra, N.H., Christofides, P.D., 2006. Stabilization of nonlinear systems with state and control constraints using Lyapunov-based predictive control. Systems \& Control Letters 55, 650-659. 
Müller, M.A., Allgöwer, F., 2012. Robustness of steady-state optimality in economic model predictive control, in: Proceedings of the 51st IEEE Conference on Decision and Control, Maui, Hawaii. pp. 1011-1016.

Müller, M.A., Angeli, D., Allgöwer, F., 2014a. On the performance of economic model predictive control with self-tuning terminal cost. Journal of Process Control 24, 1179-1186.

Müller, M.A., Angeli, D., Allgöwer, F., 2014b. Transient average constraints in economic model predictive control. Automatica 50, 2943-2950.

Omell, B.P., Chmielewski, D.J., 2013. IGCC power plant dispatch using infinitehorizon economic model predictive control. Industrial \& Engineering Chemistry Research 52, 3151-3164.

Muñoz de la Peña, D., Christofides, P.D., 2008. Lyapunov-based model predictive control of nonlinear systems subject to data losses. IEEE Transactions on Automatic Control 53, 2076-2089.

Pontryagin, L.S., Boltyanskii, V.G., Gamkrelidze, R.V., Mishchenko, E.F., 1961. Mathematical Theory of Optimal Processes. Fizmatgiz, Moscow.

Rawlings, J.B., Amrit, R., 2009. Optimizing process economic performance using model predictive control, in: Magni, L., Raimondo, D.M., Allgöwer, F. (Eds.), Nonlinear Model Predictive Control. Springer Berlin Heidelberg. volume 384 of Lecture Notes in Control and Information Sciences, pp. 119-138. 
Rawlings, J.B., Angeli, D., Bates, C.N., 2012. Fundamentals of economic model predictive control, in: Proceedings of the 51st IEEE Conference on Decision and Control, Maui, Hawaii. pp. 3851-3861.

Sontag, E.D., 1998. Mathematical Control Theory: Deterministic Finite Dimensional Systems. volume 6. Springer.

Würth, L., Marquardt, W., 2014. Infinite-horizon continuous-time NMPC via time transformation. IEEE Transactions on Automatic Control 59, 2543-2548.

Zavala, V.M., 2015. A multiobjective optimization perspective on the stability of economic MPC. Technical Report. Argonne National Laboratory.

Zhang, J., Liu, S., Liu, J., 2014. Economic model predictive control with triggered evaluations: State and output feedback. Journal of Process Control 24, $1197-$ 1206. 